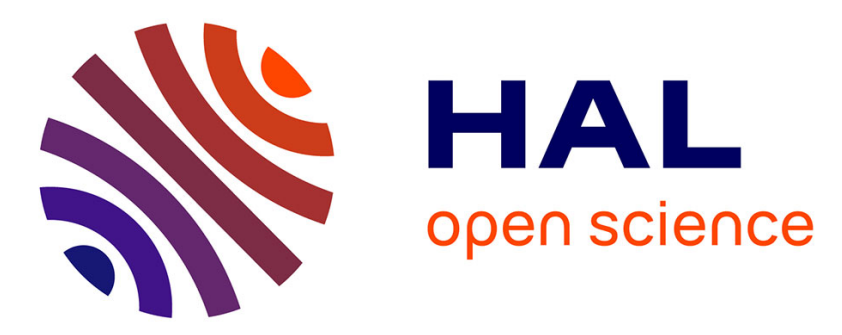

\title{
Minimizing the Effects of Navigation Uncertainties on the Spacecraft Rendezvous Precision
}

\author{
Georgia Deaconu, Christophe Louembet, Alain Théron
}

\section{To cite this version:}

Georgia Deaconu, Christophe Louembet, Alain Théron. Minimizing the Effects of Navigation Uncertainties on the Spacecraft Rendezvous Precision. Journal of Guidance, Control, and Dynamics, 2014, 37 (2), pp.695-700. 10.2514/1.62219 . hal-01078527

\section{HAL Id: hal-01078527 \\ https://hal.science/hal-01078527}

Submitted on 29 Oct 2014

HAL is a multi-disciplinary open access archive for the deposit and dissemination of scientific research documents, whether they are published or not. The documents may come from teaching and research institutions in France or abroad, or from public or private research centers.
L'archive ouverte pluridisciplinaire HAL, est destinée au dépôt et à la diffusion de documents scientifiques de niveau recherche, publiés ou non, émanant des établissements d'enseignement et de recherche français ou étrangers, des laboratoires publics ou privés. 


\title{
Minimizing the effects of navigation uncertainties on
}

\section{the spacecraft rendezvous precision}

\author{
Georgia Deaconu ${ }^{1}$ and Christophe Louembet ${ }^{2}$ and Alain Théron ${ }^{3}$ \\ CNRS; LAAS; 7 avenue du colonel Roche, F-31400 Toulouse; France \\ Univ de Toulouse; UPS, LAAS; F-31400 Toulouse; France
}

\section{Introduction}

The ability to robustly and precisely control the spacecraft relative motion will play an important role in future on-orbit inspection and on-orbit servicing missions [1]. Model Predictive Control (MPC) is considered to be an effective control strategy for these types of spacecraft operations, that can easily handle mission specific constraints while explicitly minimizing the fuel consumption [2]. The maneuvers plan is obtained by solving a finite horizon open-loop optimal control problem starting from the spacecraft relative state and the optimal solution consists of a series of control actions $\left\{u_{1}, u_{2}, \ldots, u_{N}\right\}$, out of which only the first one is executed [3].

The MPC strategies are inherently robust to arbitrarily small perturbations [4] and new measurement information is included every time the maneuvers plan is re-computed. However, in the case of spacecraft relative motion, navigation uncertainties and orbital perturbations can cause the real relative trajectory to differ significantly from the prediction used for obtaining the control plan. Reference [5] shows that small errors in the estimation of the spacecraft relative velocity can result in very large prediction errors for the relative state. Since trajectory planning relies heavily on the knowledge of the relative state, not accounting for navigation errors may have some undesirable effects such as poor performances, constraints violations and/or infeasibility of the control problem.

For circular reference orbits, a method for constraints tightening in the MPC problem was

\footnotetext{
${ }^{1}$ Methods and Algorithms in Control, LAAS-CNRS, gdeaconu@laas.fr

${ }^{2}$ Methods and Algorithms in Control, LAAS-CNRS, christophe.louembet@laas.fr

3 Methods and Algorithms in Control, LAAS-CNRS, atheron@laas.fr
} 
presented in $[6,7]$. The method uses a precomputed static feedback gain that makes the system nilpotent in at most $N$ steps. It guarantees constraints satisfaction and recursive feasibility of the optimisation problem, even when sensor noise is considered. The method however is not applicable for eccentric reference orbits, where the spacecraft relative dynamics are Linear Time Varying (LTV).

The presence of unknown but bounded navigation errors in the case of eccentric reference orbits is dealt with in [8] by propagating the uncertainties set over the prediction horizon and tightening the constraints to account for their effects. A similar idea is used in [9], combined with an online estimation of the uncertainties bounds. Reference [10] identifies the disturbance sequence that can cause the maximum variation of the spacecraft relative state and then uses this sequence to tighten the constraints of a deterministic MPC spacecraft trajectory control problem. These types of methods do provide more accurate information about the evolution of the system, but they have no control over the spread of the predicted trajectories. Moreover, tightening constraints using the open-loop propagation of the uncertainties imposes the choice of short prediction horizons in order to ensure the feasibility of the problem.

A method that directly optimizes the final spacecraft rendezvous precision without restricting the duration of the maneuvers is proposed in this article. The method is based on the so-called feedback MPC [11], which uses a sequence of feedback policies $\left\{\mu_{1}(\cdot), \ldots, \mu_{N-1}(\cdot)\right\}$ as decision variables, instead of a sequence of control actions $\left\{u_{1}, \ldots, u_{N-1}\right\}$. The computation of such feedback laws can be extremely difficult in the general case since the decision variables are infinite dimensional [12]. However, restricting the admissible feedback policies to the class of affine state feedback control laws can reduce the complexity of the problem.

For circular reference orbits, a static feedback term combined with the nominal optimal solution to the classical MPC problem could ensure that, in presence of sensing noise, all possible spacecraft relative trajectories remain inside a tube centered around the nominal trajectory [12]. When eccentric reference orbits are considered, time-varying feedback policies need to be computed in the same time as the nominal control and the problem may become non-convex. However, re-parametrising the control as affine disturbance feedback policies can remove this issue [13].

Some ideas from tube-based MPC are used in this paper to solve the robust fixed-time spacecraft 
rendezvous problem for eccentric reference orbits. The purpose is to obtain a sequence of feedback policies that steers the spacecraft from an initial relative state towards an ellipsoidal set centred around a desired final state, in presence of navigation uncertainties. This must be done while respecting the actuators saturation constraints and while pursuing a double objective: minimise the fuel cost of the mission and minimise the size of the arrival set to guarantee a good rendezvous precision. The control policies are restricted to affine disturbance feedback policies to ensure a convex formulation of the control synthesis problem. The obtained sequence of feedback policies drives the system to the guaranteed arrival set without any need for recurrent optimization.

\section{Spacecraft relative dynamics}

The fixed-time rendezvous between two spacecraft on arbitrary elliptical orbits consists of bringing the system from an initial state $X_{1}$ to a desired final state $X_{f}$ at a specified time. This needs to be achieved by firing the thrusters a fixed number of times $N$ at some predefined instants. Our goal is to determine a sequence of feedback policies that guarantees the best final rendezvous precision in presence of navigation uncertainties, while minimising the mission's fuel cost and robustly satisfying the actuators saturation constraints.

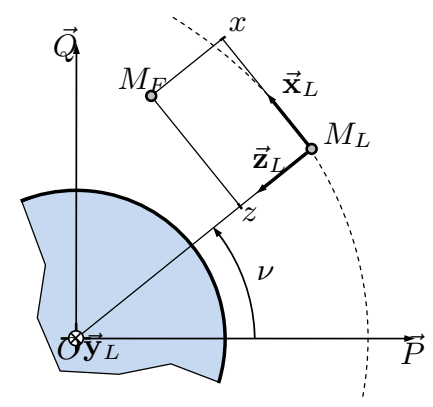

Fig. 1: The local Cartesian frame attached to the leader spacecraft

The relative state between the two spacecraft is defined as the relative position and the relative velocity, expressed in a local Cartesian frame (Figure 1) attached to the leader spacecraft $X \in \mathbb{R}^{6}$, $X=\left[\begin{array}{llllll}x & y & z & v_{x} & v_{y} & v_{z}\end{array}\right]^{T}$. The following operation can be used to transform the time domain state 
variables into scaled state variables depending on the true anomaly of the leader $\nu$ :

$$
\tilde{X}(\nu)=\left[\begin{array}{cc}
(1+e \cos \nu) I_{3} & 0_{3} \\
-e \sin \nu I_{3} & \sqrt{\frac{a^{3}\left(1-e^{2}\right)^{3}}{\mu}} \frac{1}{1+e \cos \nu} I_{3}
\end{array}\right] X(t)
$$

where $\mu$ is the Earth's gravitational parameter, $e$ is the eccentricity of the orbit of the leader spacecraft and $a$ its semi-major axis. The variable change (1) has been used by Tschauner and Hempel in [14] to obtain linearised spacecraft relative dynamics valid for arbitrary values of the eccentricity. Yamanaka and Ankersen presented in [15] a closed form solution for the TschaunerHempel equations under the form of a transition matrix $\Phi \in \mathbb{R}^{6 \times 6}$. This transition matrix is used here to propagate the spacecraft relative trajectory without numerical integration. The spacecraft relative dynamics under impulsive control $\Delta v \in \mathbb{R}^{3}$ can be written as:

$$
\tilde{X}\left(\nu_{k+1}\right)=\Phi\left(\nu_{k+1}, \nu_{k}\right)\left(\tilde{X}\left(\nu_{k}\right)+B \Delta v_{k}\right)
$$

where the matrix $B=\left[\begin{array}{ll}0_{3} & I_{3}\end{array}\right]^{T}$ models the instantaneous relative velocity change caused by the impulsive control $\Delta v_{k}$. The spacecraft relative dynamics in (2) can also be seen as an LTV system defined as:

$$
x_{k+1}=A_{k} x_{k}+B_{k} u_{k}
$$

where $x_{k}=\tilde{X}\left(\nu_{k}\right), A_{k}=\Phi\left(\nu_{k+1}, \nu_{k}\right), B_{k}=\Phi\left(\nu_{k+1}, \nu_{k}\right) B$ and $u_{k}=\Delta v_{k}$. This description of the relative dynamics will be used next to write the robust fixed-time spacecraft rendezvous problem.

\section{Disturbance feedback control}

Consider the spacecraft relative dynamics in (3) where the knowledge of the state is affected at each instant $k$ by unknown navigation errors $\delta x_{k}$. The navigation errors $\delta x_{k}$ belong to some ellipsoidal sets $E\left(0, Q_{k}\right)=\left\{x \in \mathbb{R}^{6} \mid x^{T} Q_{k} x \leq 1\right\}$. Note that the defining matrices $Q_{k} \in \mathbb{R}^{6 \times 6}$ are not necessarily identical. The real spacecraft relative state $x_{k}$ is unknown but at each step is connected to the measured state $x_{k}^{m}$ through:

$$
x_{k}=x_{k}^{m}+\delta x_{k}, \delta x_{k} \in E\left(0, Q_{k}\right)
$$

The prediction of the state that will be measured at the next step $x_{k \mid k+1}^{m}$ is given by:

$$
x_{k \mid k+1}^{m}=x_{k+1}-\delta x_{k+1}=A_{k} x_{k}+B_{k} u_{k}-\delta x_{k+1}, \delta x_{k+1} \in E\left(0, Q_{k+1}\right)
$$


Using (4) to replace the unknown state of the system with the current measure leads to:

$$
x_{k \mid k+1}^{m}=A_{k} x_{k}^{m}+B_{k} u_{k}+A_{k} \delta x_{k}-\delta x_{k+1}
$$

Let $w_{k}$ be the total contribution of the measurement noise over one prediction step. From (6) $w_{k}$ can be defined as:

$$
w_{k}=A_{k} \delta x_{k}-\delta x_{k+1}, \delta x_{k} \in E\left(0, Q_{k}\right), \delta x_{k+1} \in E\left(0, Q_{k+1}\right)
$$

Since the navigation errors belong to ellipsoidal sets that are symmetric with respect to the origin, the domain for $w_{k}$ is defined by:

$$
w_{k} \in E\left(0, A_{k}^{-T} Q_{k} A_{k}^{-1}\right) \oplus E\left(0, Q_{k+1}\right) \subseteq E\left(0, Q_{k}^{w}\right)
$$

where $\oplus$ denotes the Minkowski sum of the two sets. The ellipsoidal set $E\left(0, Q_{k}^{w}\right)$ represents an outer approximation of the real domain and it can be computed analytically, using for instance the procedure described in [16].

Denoting $\mathbf{x}=\left[\begin{array}{llll}x_{1 \mid 2}^{m} & x_{1 \mid 3}^{m} & \ldots & x_{1 \mid N}^{m}\end{array}\right]^{T}, \mathbf{u}=\left[\begin{array}{lllll}u_{1} & u_{2} & u_{3} & \ldots & u_{N-1}\end{array}\right]^{T}$ and $\mathbf{w}=\left[\begin{array}{lllll}w_{1} & w_{2} & w_{3} & \ldots & w_{N-1}\end{array}\right]^{T}$, the evolution of the measured state over the prediction horizon is given by:

$$
\mathbf{x}=\mathbf{A} x_{1}^{m}+\mathbf{B u}+\mathbf{C w}
$$

where the matrices $\mathbf{A} \in \mathbb{R}^{6(N-1) \times 6}, \mathbf{B} \in \mathbb{R}^{6(N-1) \times 3(N-1)}, \mathbf{C} \in \mathbb{R}^{6(N-1) \times 6(N-1)}$ are defined as:

$$
\mathbf{A}=\left[\begin{array}{c}
A_{1} \\
A_{2} A_{1} \\
\vdots \\
A_{N-1} \ldots A_{1}
\end{array}\right] \mathbf{B}=\left[\begin{array}{ccccc}
B_{1} & 0 & 0 & 0 & \ldots \\
A_{2} B_{1} & B_{2} & 0 & 0 & \ldots \\
\vdots & \vdots & & \\
A_{N-1} \ldots A_{2} B_{1} & & \ldots & \ldots & B_{N-1}
\end{array}\right] \mathbf{C}=\left[\begin{array}{ccccc}
I & 0 & 0 & \ldots \\
A_{2} & I & 0 & 0 & \ldots \\
\vdots & \vdots & & \\
& & & & \\
A_{N-1} \ldots A_{2} & & A_{N-1} & I
\end{array}\right]
$$

The structure chosen for the control policies $u_{k}$ is based on the results in [13] and it consists of an impulsive component plus some disturbance feedback terms used to compensate the effects of navigation errors:

$$
u_{k}=\Delta v_{k}+\sum_{i=1}^{k-1} L_{k, i} w_{i}
$$


Past disturbance terms until $k-1$ are considered in order to use the maximum amount of available information while making sure that the control law is causal. This also ensures that the disturbances will not be propagated in open loop for longer than the interval between two consecutive controls.

Denoting $\boldsymbol{\Delta} \mathbf{v}=\left[\begin{array}{l}\Delta v_{1} \Delta v_{2} \ldots \Delta v_{N-1}\end{array}\right]^{T}$, a more compact expression can be obtained:

$$
\mathbf{u}=\mathbf{\Delta} \mathbf{v}+\mathbf{L w}
$$

where the matrix $\mathbf{L} \in \mathbb{R}^{3(N-1) \times 6(N-1)}$ is defined by:

$$
\mathbf{L}=\left[\begin{array}{ccccc}
0 & 0 & 0 & \ldots & 0 \\
L_{2,1} & 0 & 0 & \ldots & 0 \\
L_{3,1} & L_{3,2} & 0 & \ldots & 0 \\
\vdots & & & & \\
L_{N-1,1} & \ldots & \ldots & L_{N-1, N-2} & 0
\end{array}\right]
$$

The spacecraft closed-loop relative dynamics can be written as:

$$
\mathbf{x}=\mathbf{A} x_{1}^{m}+\mathbf{B} \boldsymbol{\Delta} \mathbf{v}+(\mathbf{B L}+\mathbf{C}) \mathbf{w}
$$

Let $\overline{\mathbf{x}}=\left[\begin{array}{llll}\bar{x}_{1 \mid 2} & \bar{x}_{1 \mid 3} & \ldots & \bar{x}_{1 \mid N}\end{array}\right]^{T}$ be the nominal trajectory obtained when assuming perfect state information and using only the impulsive part of the control:

$$
\overline{\mathbf{x}}=\mathbf{A} x_{1}^{m}+\mathbf{B} \boldsymbol{\Delta} \mathbf{v}
$$

The impulsive plan $\Delta \mathbf{v}$ must be such that the nominal trajectory satisfies the final rendezvous objective:

$$
\bar{x}_{1 \mid N}=X_{f}
$$

In this case, the error between the perturbed trajectory and the nominal trajectory can be written as a linear function of the disturbance vector $\mathbf{w}$ :

$$
\mathbf{e}=\mathbf{x}-\overline{\mathbf{x}}=(\mathbf{B L}+\mathbf{C}) \mathbf{w}
$$

The purpose is to find the impulsive plan $\boldsymbol{\Delta} \mathbf{v}$ and the correction gains matrix $\mathbf{L}$ that guarantees the smallest error at the end of the prediction horizon $e_{N}$ and the lowest fuel cost for the nominal trajectory, all while robustly satisfying the saturation constraints on the thrusters. 
Assuming that the spacecraft are equipped with thrusters rigidly mounted on the body axes, the cost function corresponding to the fuel consumption for the nominal trajectory is defined by the sum of the $\ell_{1}$-norm of the thrust vector [17]:

$$
J_{\Delta v}=\|\boldsymbol{\Delta} \mathbf{v}\|_{1}
$$

The variables $z_{k} \in \mathbf{R}^{3}$ are introduced to linearise the objective function $J_{\Delta v}[18]$ and are such that:

$$
\left|\Delta v_{k}\right| \leq z_{k}, k=1 . . N-1
$$

The actuators saturations are defined by $\left|u_{k}\right| \leq u_{\max }$ where $u_{k}$ is defined by (11). The constraints must hold for all admissible values of the disturbances $w \in E\left(0, Q^{w}\right)$. This can be written as row-wise conic constraints on $z_{k}$ that account for the presence of the correction terms:

$$
z_{k} \leq u_{\max }-\sum_{i=1}^{k-1}\left\|L_{k, i} P_{i}^{w}\right\|_{2}, k=1 . . N-1 \text { where } P_{i}^{w}=\left(Q_{i}^{w}\right)^{-1 / 2}
$$

The objective for the final error $e_{N}$ translates into computing the smallest ellipsoidal set $E\left(0, Q_{f}^{-1}\right)$ that bounds $e_{N}$ for all admissible values of the disturbances w:

$$
\min \operatorname{tr}\left(Q_{f}\right) \text { s.t. } e_{N}^{T} Q_{f}^{-1} e_{N} \leq 1, \forall w_{i} \in E\left(0, Q_{i}^{w}\right), i=1 . . N-1
$$

Minimizing the trace of $Q_{f}$ corresponds to minimizing the sum of squares of the semi-axis of the ellipsoidal set $E\left(0, Q_{f}^{-1}\right)$. From (17), the quadratic constraint on $e_{N}$ can be written as:

$$
\mathbf{w}^{T}\left(\mathbf{B}_{\mathbf{N}} \mathbf{L}+\mathbf{C}_{\mathbf{N}}\right)^{T} Q_{f}^{-1}\left(\mathbf{B}_{\mathbf{N}} \mathbf{L}+\mathbf{C}_{\mathbf{N}}\right) \mathbf{w} \leq 1, \forall w_{i} \in E\left(0, Q_{i}^{w}\right), i=1 . . N-1
$$

where $\mathbf{B}_{\mathbf{N}}$ and $\mathbf{C}_{\mathbf{N}}$ are obtained by selecting the appropriate lines in the $\mathbf{B}$ and $\mathbf{C}$ matrices. Using the S-procedure [19] and the Schur complement, constraint (22) can be transformed into a linear matrix inequality:

$$
\begin{aligned}
& \exists \tau_{1}, \tau_{2}, \ldots \tau_{N-1} \geq 0, Q_{f} \geq 0 \\
& {\left[\begin{array}{ccc}
1-\sum_{k=1}^{N-1} \tau_{k} & 0 & 0 \\
0 & Q_{w} & \left(\mathbf{B}_{\mathbf{N}} \mathbf{L}+\mathbf{C}_{\mathbf{N}}\right)^{T} \\
0 & \left(\mathbf{B}_{\mathbf{N}} \mathbf{L}+\mathbf{C}_{\mathbf{N}}\right) & Q_{f}
\end{array}\right] \geq 0, \quad Q_{w}=\left[\begin{array}{ccc}
\tau_{1} Q_{1}^{w} & & \\
& \ddots & \\
& & \tau_{N-1} Q_{N-1}^{w}
\end{array}\right]}
\end{aligned}
$$

The conic optimization problem that must finally be solved is given by:

$$
\begin{array}{ll}
\min _{Q_{f}, z_{k}, \Delta v_{k}, \mathbf{L}} & \operatorname{tr}\left(Q_{f}\right)+\sum_{k=1}^{N-1} z_{k} \\
\text { s.t. } & (16),(19),(20),(23)
\end{array}
$$


The structure chosen for the control $u_{k}$, other than leading to a convex optimization problem, also presents the advantage that the corrections for the effects of previous measurement errors are made in the same time as the burns for the nominal trajectory. This ensures that the total number of maneuvers remains constant and that no extra stress is put on the thrusters.

\section{Simulation results}

The fixed-time rendezvous scenarios summarized in Table 1 and 2 are used to illustrate the effectiveness of disturbance feedback control in solving this type of problem. The maneuvers plan is computed based on the linearised spacecraft dynamics and then tested on an industrial non-linear simulator. The initial spacecraft relative state used in the simulations is obtained by adding random noise to the initial state used for control computation. Random noise is also added to every other state measure and the magnitude of the noise is bounded by a $0.02 \mathrm{~m}$ ellipsoidal set for position and $0.002 \mathrm{~m} / \mathrm{s}$ for velocity.

The control $u_{k}$ is obtained from (11), where $\boldsymbol{\Delta} \mathbf{v}$ and $\mathbf{L}$ have been obtained by solving (24) before the simulation. The disturbance terms $w$ are estimated during the closed loop simulations using the perturbed state measures and (6) and (7), : $w_{k-1}=x_{k}^{m}-A_{k-1} x_{k-1}^{m}-B_{k-1} u_{k-1}$.

Table 1: Prisma mission simulation data

\begin{tabular}{cccccccccc}
\hline \hline \multicolumn{1}{c}{$e$} & $a[\mathrm{~km}]$ & $\mathrm{i}\left[{ }^{\circ}\right]$ & $\Omega\left[{ }^{\circ}\right]$ & $\omega\left[{ }^{\circ}\right]$ & $\nu_{1}\left[{ }^{\circ}\right]$ & $X_{1}[\mathrm{~m}, \mathrm{~m} / \mathrm{s}]$ & $X_{f}[\mathrm{~m}, \mathrm{~m} / \mathrm{s}]$ & $\operatorname{duration}[\mathrm{s}]$ & $u_{\max }[\mathrm{m} / \mathrm{s}]$ \\
\hline 0.004 & 7011 & 98 & 190 & 0 & 0 & {$[10000,0,0,0,0,0]$} & {$[330,0,30,0,0,-0.0158]$} & 18000 & 0.26 \\
\hline \hline
\end{tabular}

Table 2: Simbol X mission simulation data

\begin{tabular}{cccccccccc}
\hline \hline \multicolumn{1}{c}{$e$} & $a[\mathrm{~km}]$ & $\mathrm{i}\left[{ }^{\circ}\right]$ & $\Omega\left[^{\circ}\right]$ & $\omega\left[^{\circ}\right]$ & $\nu_{1}\left[{ }^{\circ}\right]$ & $X_{1}[\mathrm{~m}, \mathrm{~m} / \mathrm{s}]$ & $X_{f}[\mathrm{~m}, \mathrm{~m} / \mathrm{s}]$ & $\operatorname{duration}[\mathrm{s}]$ & $u_{\max }[\mathrm{m} / \mathrm{s}]$ \\
\hline 0.7988 & 106246.975 & 5.2 & 180 & 90 & 135 & {$[-305,0,396,0,0,0]$} & {$[-60.2,0,79.85,0,0,0]$} & 8000 & 0.8 \\
\hline \hline
\end{tabular}

First, the dimension of the guaranteed ellipsoidal arrival set defined by the matrix $Q_{f}$ is compared against the estimation provided by a classical MPC approach based on the open-loop propagation of the state uncertainty [8]. The results obtained for the two missions using $N=10$ control 
instants and for different rendezvous durations are given in Tables 3 and 4 respectively. For the Prisma mission, the disturbance feedback control method guarantees in every case a smaller arrival set if the sum of the semi-axes is considered. For the Simbol X mission, the duration of the mission is a lot smaller than the orbital period of the leader. In this case, the estimates provided by the disturbance MPC method are larger for shorter horizons but this changes as the length of the duration of the mission increases.

Table 3: Semi-axes of the arrival set in the $x z$ plane for the Prisma mission and $N=10$

\begin{tabular}{|c|c|c|c|c|c|c|c|c|}
\hline mission duration $[\mathrm{s}]$ & \multicolumn{2}{|c|}{6000} & \multicolumn{2}{|c|}{9000} & \multicolumn{2}{|c|}{12000} & \multicolumn{2}{|c|}{18000} \\
\hline disturbance feedback MPC $[\mathrm{m}]$ & 3.66 & 2.83 & 5.77 & 3.53 & 8.76 & 4.31 & 17.49 & 5.41 \\
\hline open-loop MPC [m] & 35.03 & 0.31 & 56.82 & 1.4 & 70.08 & 0.61 & 105.2 & 0.9 \\
\hline
\end{tabular}

Table 4: Semi-axes of the arrival set in the $x z$ plane for the Simbol X mission and $N=10$

\begin{tabular}{c|rr|rr|rr|rr}
\hline \hline mission duration [s] & \multicolumn{2}{|c|}{8000} & \multicolumn{2}{c|}{12000} & \multicolumn{2}{c}{16000} & \multicolumn{2}{c}{20000} \\
\hline disturbance feedback MPC [m] & 22.61 & 22.61 & 26.51 & 26.51 & 29.02 & 29.02 & 31.34 & 31.33 \\
open-loop MPC [m] & 16.15 & 15.92 & 24.48 & 23.75 & 33.04 & 31.5 & 41.86 & 39.08 \\
\hline \hline
\end{tabular}

It should be noted that while the size of the arrival set for the open-loop MPC depends solely on the duration of the mission, the performances of the disturbance feedback scheme also depend on the number of control instants. The trajectories obtained for the Prisma rendezvous mission when using the disturbance feedback control technique with $N=6$ and $N=10$ control instants are presented in Figure 2. Fifty perturbed initial conditions are considered and the control is applied without any re-computation of the impulsive part or of the correction gains. The errors with respect to the desired final position $X_{f}$ belong each time to the ellipsoidal arrival set defined by the matrix $Q_{f}$, as guaranteed by the algorithm. The dimensions of the estimated arrival set are bigger when $N=6$, reflecting the fact that the interval between two consecutive controls is larger $(3600 \mathrm{~s}$ with respect to $2000 \mathrm{~s}$ for $N=10)$.

Figure 3 presents the closed loop trajectories obtained for the Simbol X rendezvous mission. 

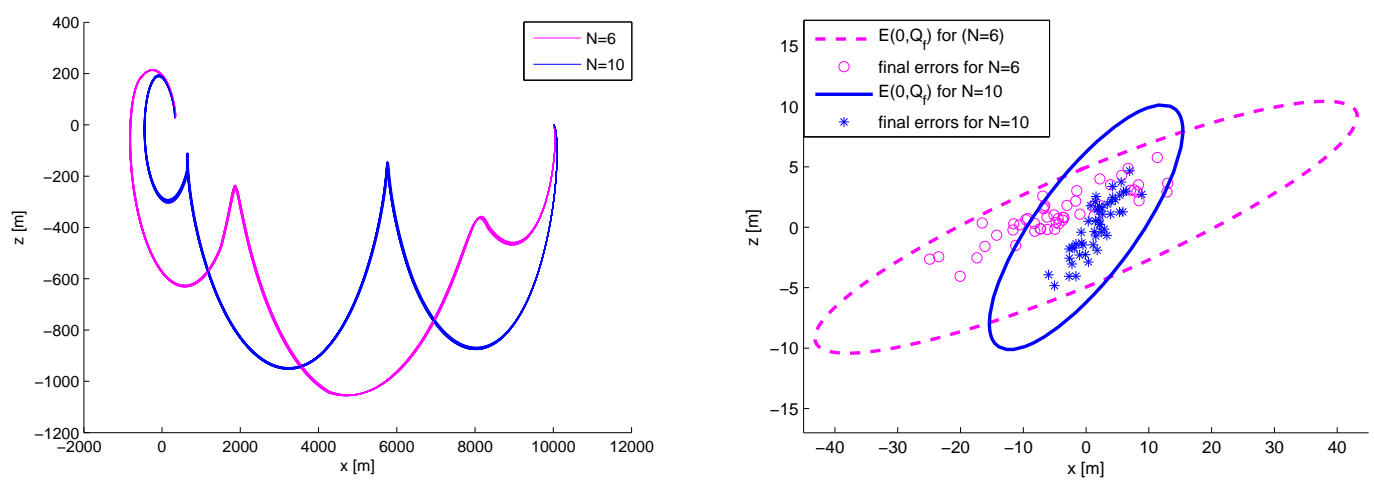

Fig. 2: Closed-loop trajectories and final errors for the Prisma mission

The final errors are all contained inside the guaranteed arrival set, but for this mission where the reference orbit is highly eccentric the estimation given for the final set seems to be more conservative.
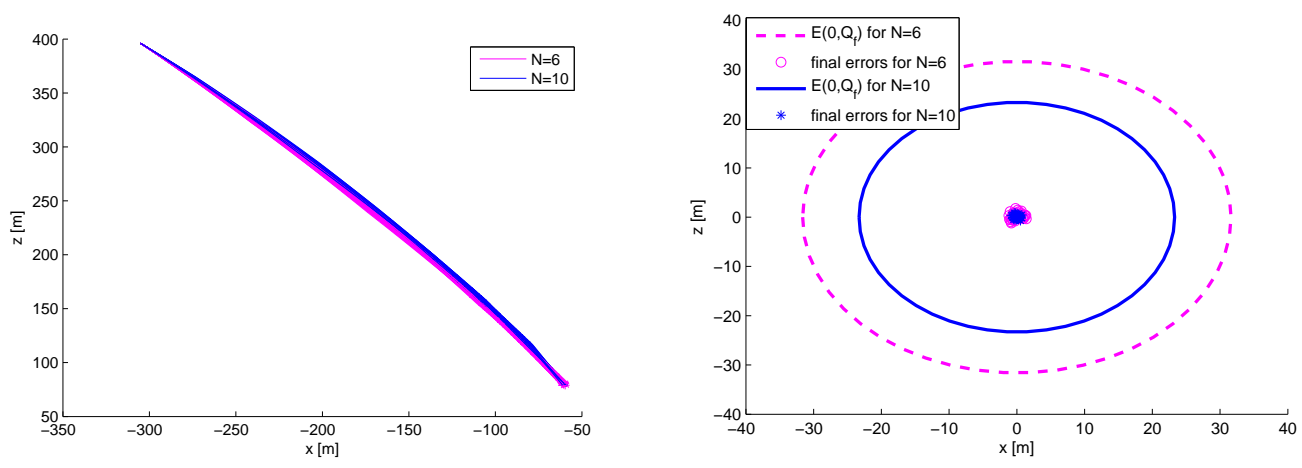

Fig. 3: Closed-loop trajectories and final errors for the Simbol X mission

\section{Conclusion}

The article shows that the disturbance feedback Model Predictive Control (MPC) can be used for effectively solving the fixed time rendezvous problem in presence of navigation uncertainties. It provides several advantages over the classical MPC approaches such as better a priori guarantees for the closed-loop system behaviour for any admissible value of the uncertainty. It also avoids problems linked to infeasibility when the satellites are in close range since it does not rely on repeated re-computations to achieve robustness. If a feasible solution is found, the rendezvous maneuvers plan can be applied without modifications, at the cost of only some simple algebraic computations for the on-line estimation of the disturbance terms. The plan can be computed by the 
ground control station and then uploaded to the satellite. The corrections accounting for the effects

of the navigation uncertainties are made in the same time as the burns for the nominal trajectory thus keeping the number of firing of the thrusters constant.

\section{References}

[1] Saleh, J., Hastings, D., Lamassoure, E., and Newman, D., "Flexibility and the Value of On-Orbit Servicing: New Customer-Centric Perspective," Journal of Spacecraft and Rockets, Vol. 40, No. 2, 2003, pp. 279-291.

[2] Di Cairano, S., Park, H., and Kolmanovsky, I., "Model Predictive Control approach for guidance of spacecraft rendezvous and proximity maneuvering," International Journal of Robust and Nonlinear Control, Vol. 22, 2012, pp. 1398-1427.

[3] Mayne, D. Q., Rawlings, J. B., Rao, C. V., and Scokaert, P. O. M., "Constrained model predictive control : Stability and optimality," Automatica, Vol. 36, 2000, pp. 789-814.

[4] Grimm, G., Messina, M., Tuna, S., and Teel, A., "Nominally Robust Model Predictive Control With State Constraints," IEEE Transactions on Automatic Control, Vol. 52, No. 10, 2007, pp. 1856-1870.

[5] How, J. and Tillerson, M., "Analysis of the impact of sensor noise on formation flying control," American Control Conference (ACC), Vol. 5, 2001, pp. 3986-3991.

[6] Richards, A. and How, J., "Analytical Performance Prediction for Robust Constrained Model Predictive Control," AIAA Guidance, Navigation and Control Conference and Exhibit, No. August, 2004, pp. AIAA 2004-5110.

[7] Breger, L., How, J., and Richards, A., "Model predictive control of spacecraft formations with sensing noise," American Control Conference (ACC), 2005, pp. 2385-2390.

[8] Louembet, C., Arzelier, D., Deaconu, G., and Blanc-Paques, P., "Robust rendezvous planning under navigation and maneuvering errors," International ESA Conference on Guidance and Navigation Control Systems, 2011.

[9] Gavilan, F., Vazquez, R., and Camacho, E., "Robust Model Predictive Control for Spacecraft Rendezvous with Online Prediction of Disturbance Bounds," IFAC Workshop on Aerospace Guidance, Navigation and Flight Control Systems, 2009.

[10] Breger, L., Inalhan, G., Tillerson, M., and How, J., "Cooperative Spacecraft Formation Flying: Model Predictive Control with Open- and Closed-Loop Robustness," Modern Astrodynamics, edited by P. Gurfil, chap. 8, Elsevier Academic Press, 2007, pp. 237-277. 
[11] Langson, W., Chryssochoos, I., Rakovic, S., and Mayne, D. Q., "Robust model predictive control using tubes," Automatica, Vol. 40, No. 1, 2004, pp. 125-133.

[12] Mayne, D. Q., Kerrigan, E., and Falugi, P., "Robust model predictive control : advantages and disadvantages of tube-based methods," 18th IFAC World Congress, Milano, 2011, pp. 191-196.

[13] Goulart, P., Kerrigan, E., and Maciejowski, J., "Optimization over state feedback policies for robust control with constraints," Automatica, Vol. 42, No. 4, April 2006, pp. 523-533.

[14] Tschauner, J., "Elliptic orbit rendezvous," AIAA Journal, Vol. 5, No. 6, 1967, pp. 1110-1113.

[15] Yamanaka, K. and Ankersen, F., "New state transition matrix for relative motion on an arbitrary elliptical orbit," Journal of Guidance, Control, and Dynamics, Vol. 25, No. 1, 2002, pp. 60-66.

[16] Ros, L., Sabater, A., and Thomas, F., "An ellipsoidal calculus based on propagation and fusion." IEEE transactions on systems, man, and cybernetics. Part B: Cybernetics, Vol. 32, No. 4, 2002, pp. 430-42.

[17] Ross, I., "Space Trajectory Optimization and L1-Optimal Control Problems," Modern Astrodynamics, edited by P. Gurfil, chap. 6, Elsevier Academic Press, 2007, pp. 155-188.

[18] Bertsimas, D. and Tsitsiklis, J., Introduction to Linear Optimization, Athena Scientific, 1997.

[19] Boyd, S., El Ghaoui, L., Feron, E., and Balakrishnam, V., "Some standard problems involving LMIs," Linear Matrix Inequalities in System and Control Theory, chap. 2, SIAM, 1994, pp. 23-35. 